\title{
Streptozotocin Generates Peroxynitrite Luminescence Which Reveals the Probable Cause of Diabetes 1,2 and 3 (Alzheimer's disease)
}

\author{
Knox Van Dyke*1, Werner Geldenhuys ${ }^{2}$ and Aaron Robart ${ }^{1}$ \\ ${ }^{1}$ Departments of Biochemistry and Molecular Pharmacology, West Virginia Medical Center, Morgantown, West \\ Virginia, USA \\ ${ }^{2}$ Department of Pharmacy, West Virginia Medical Center, Morgantown, West Virginia, USA
}

Received: 23 March, 2017; Accepted: 30 July, 2017; Published: 28 August, 2017

*Corresponding author: Knox Van Dyke, Department of Biochemistry and Molecular Pharmacology, West Virginia Medical Center, Morgantown, West Virginia, USA; E-mail: kvandyke@hsc.wvu.edu

\begin{abstract}
The fundamental basis of diabetes continues to go unrecognized and untreated. Depending on the type of diabetes i.e; 1 or 2, treatment consists of insulin injections (1) or antidiabetic drugs that lower blood glucose and hopefully hemoglobin A1C as well (2) Often patients with type 2 are asked to lose weight, exercise more vigorously as well and more frequently. Previously, using a diabetic- streptozotocin (STZ) rat model, we demonstrated that diabetes and cataracts could be entirely prevented using equimolar amounts of STZ and a nitric oxide oxidizing compound called sodium carboxy-PTIO. This indicated that STZ generated a nitric oxide -oxygen based toxin that could be destroyed in vivo. The treatment completely prevented STZ from increasing blood glucose and all the signs of diabetes- including cataracts were prevented. Later work indicated how the STZ-diabetes pathway was likely created. Now, we demonstrate that STZ actually generates the oxidizing and nitrating peroxide known as peroxynitrite (OONO-). In the cases of diabetes 1,2 most or all of many anti-diabetic drugs only control the level of blood glucose, and many with considerable side effects. We demonstrate in diabetic children over a three year period that even with tight dietary control of blood glucose, diabetes still persists. This diabetic damage stems from peroxynitrite. Using STZ to generate peroxynitrite in animals, we demonstrate that a variety of nitration targets can diminish peroxynitite which is the most important step in controlling both diabetes and possibly Alzheimer's disease since recent data links diabetes and Alzheimer's disease.
\end{abstract}

\section{Introduction}

The most extensively used animal model to create either type 1 or 2 diabetes is the utilization of Injected Streptozotocin (STZ) which was originally developed as an antibiotic for gram negative bacteria by the Upjohn company of Kalamazoo, Michigan and later found to cause diabetes [1]. Depending on the dose of STZ injected- either type 1 (high dose) or type 2 (low dose) causes these diseases. It is well understood that STZ causes the death of the beta cells of the pancreas. Therefore, high doses of STZ kill most or all of the beta cells and lower doses create the killing of some beta cells which produce insulin, but spares some of the beta cells. STZ injected rats increases blood glucose from normal levels (100 milligrams /dl) to about $500 \mathrm{mg} / \mathrm{dl}$ displaying type 1 diabetes in mice or rats with blood glucose $300 \mathrm{mg} / \mathrm{dl}$ in the type 2 diabetic animals [2,3]. In a previous publication in Luminescence, we demonstrated that diabetes caused by streptozotocin could be completely blocked or abrogated using an equimolar concentration of Carboxy-PTIO (C-PTIO) and STZ [4]. Since it is known that C-PTIO oxidizes nitric oxide (.NO) to NO2, it prevents the nitric oxide from being utilized to form the nitric oxide-based toxin (OONO-) in vivo. Naturally, if the toxin cannot form, diabetes does not develop. One of the reviewer's of this original paper commented that this was the beginning of the end for diabetes and naturally we believe this comment to be quite correct.

In later work, using targets of nitration, we demonstrated that these could also prevent streptozotocin based diabetes [3,5]. The structure of STZ is 2 deoxyglucose bonded to a methylated nitrosourea (Figure1).

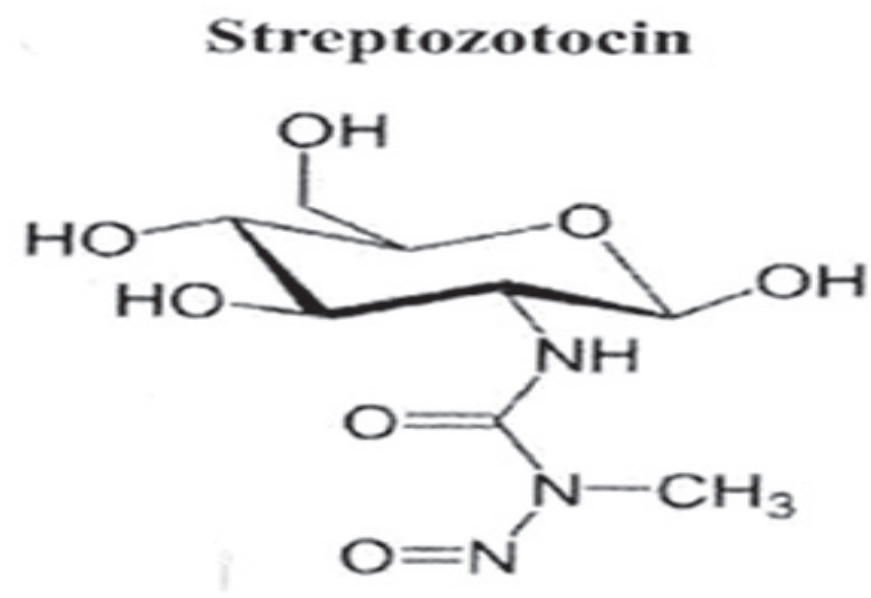

Figure 1: Structure of streptozotocin (STZ)-2 deoxyglucose attached to methylnitrosourea.

It is known that tissues that have substantial amounts of the sugar transport mechanism-( glut-2) tend to concentrate glucose type molecules like 2 deoxyglucose and be more damaged by STZ eg. beta cells of the pancreas, liver and kidney cells. Once the STZ with 2-deoxyglucose bonded to the methylated nitrosourea gets into the beta cell, the STZ is split into 2 deoxy glucose and methylated nitrosourea (MNU). The MNU 
can generate the nitric oxide free radical which reacts with superoxide (.02-) producing (OONO- or later its carbonate derivative) and MNU can methylate DNA bases, break both single stranded or double stranded DNA or even RNA, activate poly-ADP-ribose polymerase (PARP) depleting NADH and ATP killing mitochondria and cells dependent on it- and causing nitration, nitrosylation and nitrosation of key proteins and damaging their important enzymatic activity [6]. In a recent JBC paper Andersson et al., it was suggested that STZ could directly generate peroxynitrite [7]. Their evidence was that when STZ and tyrosine reacted- that di-tyrosine was produced. This is only evidence that free radicals are produced- which we already knew, since STZ can clearly generate the nitric oxide radical as was both indicated from our previous studies and studies by others Tsuji and Sakurai $[3,5,8]$.

Furthermore, a similar set of diabetic conditions of impaired glucose utilization by Chen and Zhong and insulin resistance occurs in early Alzheimer's disease [9]. This can be identified in humans using PET scanning via injection into the body of 18-fluorodeoxyglucose (FDG), a sugar which is transported into brain like glucose but whose metabolism stops after being phosphorylated, thus since this radioactive remnant remains long enough to produce superb PET scans [10]. The pattern of glucose utilization is depressed in early-stage dementia or pre-Alzheimer's disease (AD) compared to people without disease. This is almost certainly an early marker of the AD before significant loss of neurons and/or memory occurs. When streptozotocin is intra-articulary injected into mouse brain, glucose utilization in the mouse brain is depressed and insulin resistance occurs similar to when STZ is injected into rodents peripherally damaging Beta cells causing diabetes [11,12]. Further, after a few months, neurons in the brain die, and plaques and tangles occur with marked dementia developing. This is clearly the best model of Alzheimer's disease ever developed! Many investigators have clearly demonstrated that STZ injection into the brain is a superior method compared to the gene defect models in mice which do not demonstrate many of the complete characteristics of human Alzheimer's disease. It should not be too surprising that some investigators have termed Alzheimer's disease as diabetes 3 because of its similarity to diabetes [9]. Many investigators have switched to this STZ -ALZ based superior standard mouse model to study possible inhibitors of the disease Kimura and Lecanu and Papadopoulous $[13,14]$.

In this manuscript, we will develop- what we believe is clear evidence that STZ does generate a peroxide namely peroxynitrite (PN) $(\mathrm{OON}=\mathrm{O}-)$ and show that the reaction of STZ producing PN generates luminescence via its reaction with L-012. Furthermore, STZ based luminescence is abrogated by micromolar doses of acetaminophen or tyrosine hydrochloride or L-cysteine hydrochloride,etc. acting as targets of oxidation or nitration and whose nitrated or oxidized product has been confirmed by mass spectroscopy (not shown). The reaction of STZ with L-012 is very similar to the peroxynitrite generation reaction of SIN-1 (sydnoimine) with L-012, whose luminescent reaction generates similar blue luminescence reaction kinetics as we have previously published in Luminescence 2007 [15]. A compound must be very strong oxidizer to react with $\mathrm{L}-012$ to produce blue light production eg because hydrogen peroxide, hypochlorite or superoxide do not react with L-012. L-012 luminescence from peroxynitrite or streptozotocin both produce blue light which is extinguished by tyrosine. $\mathrm{HCl}$, acetaminophen or L-cysteine. $\mathrm{HCl}$ or, L-cysteine or mono or polyphenols depending on the polyphenolic structure published in Van Dyke et al. [16].

\section{Methods}

\section{Drugs, Chemicals, Materials and Equipment}

(Streptozotocin) or STZ and 4 hydroxy Tempo (4HT) as well as phosphate buffered saline or PBS ( $\mathrm{pH}=7.4)$ and carboxy-PTIO sodium salt and other L- amino acids like L-tyrosine, $\mathrm{N}$-acetyl cysteine was purchased from Sigma Chemicals (SC) Saint Louis ,MO., except where noted. L-012 was purchased from Waco Chemicals, Richmond, VA., and SIN -1 (linsidomine which generates peroxynitrite) was purchased from Cayman Chemical company- Ann Arbor Michigan. Acetaminophen was a kind gift from the McNeil Drug Company, Philadelphia, PA. Horse radish peroxidase (HRP) was obtained from the Worthington enzyme company.

\section{Luminescence Assays}

We measured luminescence using L-012 and either SIN-1 or streptozotocin both of which are peroxynitrite generators using a Berthold 6 channel luminometer model LB 9505C. All assays were done using a $3 \mathrm{ml}$ Berthold round bottom luminometer tubes using a 500 microliter total volume in each and the various ingredients dissolved in PBS added for each tube. The assays with various named ingredients discussed in Figures are accomplished at $37 \mathrm{C}$ for a period of 20 minutes. Therefore, the luminescence for the entire 20 minutes is integrated using KINB software supplied by the Berthold Company and expressed as integrated counts per minute (arbitrary units) over the reaction period. The reactions are viewed in real time on a monitor linked to the luminometer and results can be printed in color on the accompanying printer post assay. CarboxyPTIO or acetaminophen, L- Tyrosine, L-N-Acertyl cysteine were assessed for their anti-peroxynitrite luminescence activity using L-012, a luminol -like compound which generates 100 times more luminescence with peroxynitrite than similar concentrations of luminol.

\section{Blood Glucose Assay for Rats}

Specifically blood from controls or treated rats was obtained from the end of the barely snipped tail of a rat after first applying a local or topical anesthetic salve used in clinical dentistry to prevent pain of injection. A small drop of blood was applied to the edge of a glucose monitoring strip inserted into a Therasense Glucose monitoring device used for monitoring blood glucose (BG) levels in humans. It works because the monitoring strips contain glucose oxidase which reacts with the blood d-glucose creating an electric current which is proportional to the concentration of glucose and the calibrated monitor reads in milligrams of glucose/per deciliter of blood.

\section{Production of Peroxynitrite-based Luminescence from Streptozotocin and its Inhibition by Acetaminophen, L-tyrosine, and L-cysteine}

L-012 was used in all current luminescence assays. In some cases we used SIN-1 to generate peroxynitrite to generate blue luminescence, and in other cases we used to streptozotocin and, L-012, but we added a trace 
amount of horse radish peroxidase (HRP) to enhance the luminescent reaction. Streptozotocin will react with L-012 without addition of HRP, but the reaction without HRP appears to occur more slowly and less vigorously. Both SIN-1 and streptozotocin become unstable when warmed in buffer so they must be made quickly, maintained on ice and diluted prior to assay and added last when initiating a luminescent reaction. The two compounds should be kept on ice after they are dissolved in cold PBS. SIN-1 and STZ must be handled carefully-- since they are temperature sensitive when dissolved in PBS. The stock concentration of SIN-1 is $5 \mathrm{mg}$ /50 ml of cold PBS. This is diluted 1:1000 with PBS and stored on ice and added last in the order of ingredients to each tube. STZ was added to PBS at a final concentration of $1 \mathrm{mg} / \mathrm{ml}$ PBS buffer.

The L-012 in dilute solution (above) contains a trace of horse radish peroxidase (HRP) to produce enhanced luminescence more quickly. The fact the reaction is better with HRP added, indicates that a peroxide (peroxynitrite ) is reacting with the HRP. When the STZ-HRP reaction oxidizes L-012 it produces almost a million counts/minute and the blue light is inhibited by 10 micromolar acetaminophen (Tylenol) etc.. This is a similar occurrence as when SIN-1 which generates peroxynitrite oxidizes L-012 to produce blue luminescence. Both of these luminescent reactions are inhibited by Tylenol and some other amino acids in 10 micromolar doses as previously mentioned because they destroy peroxynitrite since they are nitrated or oxidized by PN thus destroying peroxynitrite's ability to react with L-012 creating inhibited luminescence.

\section{E-Gel Electrophoresis Using 2\% Agarose System by Invitrogen}

We added a $10 \%$ solution of streptozotocin to an equal volume of DNA ladder solution from Invitrogen and incubated it for 30 minutes at 37C.and compared it using the same conditions of an untreated control DNA ladder sample dilute DNA ladder diluted in running buffer. Post incubation, ten microliters of each sample was placed in different wells of the agarose cassette. Plasmid DNA (g-WIZ-luc luciferase plasmid by Aldevron (6372 base pairs) treated with streptozotocin diluted in running buffer were incubated and placed in wells as above. The cassette has SYBR Safe dye incorporated into the $2 \%$ agarose gel for staining the DNA (yellow fluorescence). The samples are subjected to the same conditions of electricity specified by electrophoresis casette for 30 minutes and examined under short wave ultraviolet light and photographed. It is noted that streptozotocin damages the plasmid but not the ladder DNA (visually). It is well known that peroxynitrite follows this same damage pattern as STZ.

\section{Drug Treatment of Animals}

Sprague Dawley rats were purchased from a local supplier and all animal work was done according to rules of the Animal Care Committee of the West Virginia University Medical School. We made handling animals with the same special care as if these were our own pets. For example, we would use surface/topical anesthesia, if we were to draw blood samples for glucose assay so the animal would receive minimal pain from the 27 gage needle. The animals in this study weighed about 300 grams. Eight rats were injected subcutaneously with a mixture of $0.5 \mathrm{ml} \mathrm{STZ}$ at $30 \mathrm{mg}$ / $\mathrm{kg}$ dissolved in PBS, $\mathrm{pH}=7.4$ with carboxy-PTIO equimolar to STZ also dissolved in PBS. Simultaneously, 6 rats were injected with STZ only at $0.5 \mathrm{ml}$ at a concentration of $30 \mathrm{mg} / \mathrm{kg}$-so they would become partially diabetic i.e; type 2 like diabetic but would not need insulin injections to stay alive nor would they lose weight during the yearly course of the experiment.. Six rats were injected with similar amounts of saline but without STZ or drugs so that they would not become diabetic and would represent normal rats.

\section{Diabetes I Studies in Children}

Diabetes studies were accomplished in 35 children with early type 1diabetes (ages were 3-12 years) and similar type children without diabetes were used as controls. These studies were done in children with parent's consent and with the permission of the West Virginia University Committee for Clinical Studies under the rules of The United States Food and Drug for clinical studies in children. Basically a few milliliters of blood was withdrawn from each child- monthly in the clinic by licensed phlebotomists and assayed via standard clinical assays for glucose and urate. The children were in a fasting condition prior to assay during the study which lasted for a period of three years. This clinical study was accomplished under the supervision of Robert Hoeldtke, MD, Ph.D.in the clinic at West Virginia University Medical School, Morgantown, WV 26506.

\section{Statistical Analysis}

All of the quantitative experiments in animals in this report were accomplished four or more times and the means and standard error of the mean (SEM) for like experiments were calculated. In all of these data, no overlap between like groups in multiple experments was ever observed. The statistical analysis between important groups eg., STZ only, STZ/CPTIO or saline only were $>95 \%$ confidence level due to the completely separated differences between the data from different groups, and little variation existed within the groups. The experimental STZ group developed clear cut diabetes overnight, as measured by blood glucose levels (about $300 \mathrm{mg} / \mathrm{dl}$-compared to $100 \mathrm{mg} / \mathrm{dl}$ for normal rats) while the STZ/CPTIO group never developed increased blood glucose levels nor cataracts in their lenses 3 months post STZ injection as occurred with animals injected with STZ alone. CPTIO completely protected against STZ toxicity and the glucose level of these CPTIO protected animals stayed normal for longer than a year and the experiment was terminated.

\section{Results and Discussion}

\section{Preventing Diabetes and Cataracts}

In figure 2 is displayed the amazing effect of carboxy-PTIO on streptozotocin- injected animals since this drug is well known to oxidize nitric oxide producing NO2 or nitrogen dioxide. Why would this prevent the damage from streptozotocin which is a known toxin to the pancreatic beta cells causing diabetes? Why would it prevent cataracts which are known to be caused by diabetes? As we will later demonstrate streptozotocin acts as a Trojan horse in the beta cells (Figure 3). We know that the deoxyglucose portion of STZ causes it to enter cells and concentrate in cells that have the glut 2 sugar transport mechanism. The methyl nitrosourea portion of STZ separates from the deoxy glucose and causes methylation of DNA bases and generates both nitric oxide and superoxide generating peroxynitrite causing all the six different pathologies associated with diabetes as well 


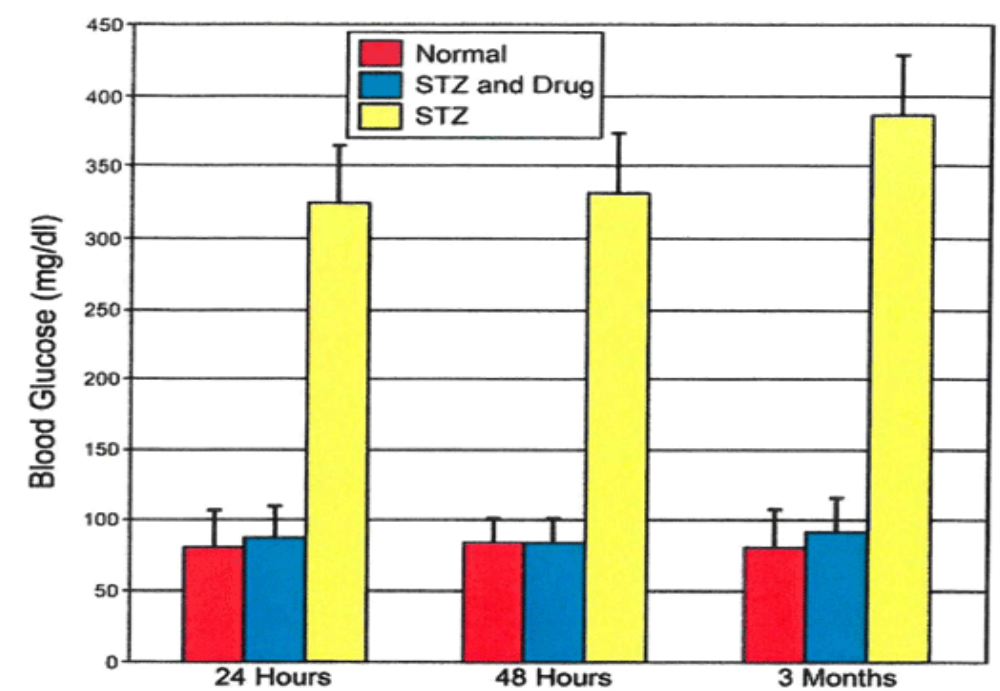

Figure 2: Effect of carboxy-PTIO (C-PTIO) and acetaminophen (Tylenol) on blood glucose levels of rats injected with a medium concentration of STZ (30 $\mathrm{mg} / \mathrm{kg}$ ) or no STZ. The concentration of C-PTIO and STZ were equimolar and the dose of Tylenol was $30 \mathrm{mg} / \mathrm{rat}$. All given subcutaneously. as the denaturation of the crystalline proteins that compose the lens of the eye which causes clouding of the lens known as cataracts [6]. Cataract surgery is the number one most common surgery in the United States. By controlling peroxynitrite production in the eye, we could prevent the need for cataract surgery. The apparent sequence of events in streptozotocin based animal diabetes and likely human diabetes as well since we know that type 1 diabetes can be controlled by inhibiting macrophage activity which is the main generator of peroxynitrite associated with beta cells.

Production of excessive amounts of nitric oxide and superoxide emanate from STZ by generating peroxynitrite inside the Beta cells of the pancreas causes the death of the Beta cell. Since peroxynitrite is an oxidizing and nitrating chemical substance- when it is made in excessive amounts in a continuous manner, the chemical damage it causes continues for a long time [17].
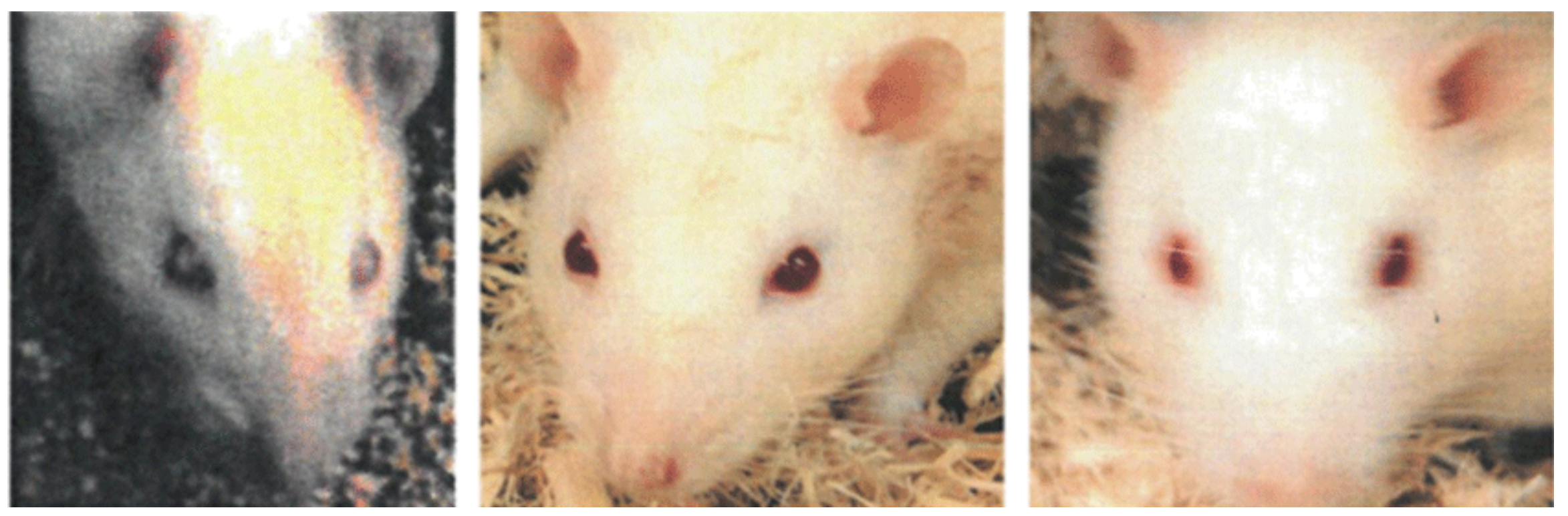

Figure 3: Picture of rats after 3 months post injection of SZ,STZ and C-PTIO/acetaminophen combination or saline injection only. The STZ animal has cataracts and the saline or drug protected animal have no cataracts.

Peroxynitrite attacks and damages DNA, RNA, proteins and lipids. DNA is damaged and knicked and/ or mutated and the damage causes activation of the DNA repair PARP or poly-ADP-ribose polymerase which uses excessive amounts of NADH and ATP killing the mitochondria and killing the beta cells via necrosis. The damaged DNA is released from dead cells along with small molecules like urate or ATP which stimulates inflammasomes inside inflammatory cells to produce and release inflammatory cell products, for example prostaglandins, interleukins, and tumor necrosis factor alpha, chemokines, nitric oxide and superoxide etc. with stimulation of key inflammatory receptors of key inflammatory and other cells $(17,18)$.

How could we interdict in the diabetic-damage process to assist insulin and other anti-diabetes drugs creating a less harmful diabetic state? We would need to do two different things to properly control the diabetic state whether treating either type 1 or 2 diabetes. The first one is to treat excessive blood glucose levels by using insulin or other anti-diabetic drugs, or controlling diet via low ingestion of glucose or fat generating substances with proper exercising and the second one is controlling excessive generation of peroxynitrite, which is certainly the major toxin causing diabetes. See figures 4 and 5 which explain the pathway causing diabetes and a possible general treatment.. We noticed that each and every year of a 3 year period that blood urate was consistently $25 \%$ lower each and every year in young diabetic children with type 1 diabetes(whether blood glucose was tightly or poorly regulated) compared to normal control children over that same period (studied in 37 children) (See Figure 6). This observation clearly demonstrates that simply controlling glucose poorly or well still creates a lowering of our most important antioxidant (urate) in the serum which is responsible for about $2 / 3$ of the antioxidant capacity of the blood serum. We believe this is a clear and unmistakable observation which indicates that it is necessary to control excessive oxidation/nitration caused by peroxynitrite to prevent continuous damage caused by the diabetic state unrelated to damage caused by excessive glucose levels. We must control excessive peroxynitrite because it is the root cause of diabetes itself, regardless if it is type 1 or type 2 diabetes.

Why do we believe that streptozotocin generates peroxynitrite? We have done many experiments which demonstrate that streptozotocin can oxidize L-012 to produce blue luminescence. L-012 will not react with superoxide, nitric oxide, hydrogen peroxide or hypochlorite. It

Citation: Dyke KV, Robart A, et al. (2017) Streptozotocin Generates Peroxynitrite Luminescence Which Reveals the Probable Cause of Diabetes 1,2 and 3 (Alzheimer's disease) Int J Open Access Ophthal 2(2):1-8. DOI: http://dx.doi.org/10.15226/2474-9249/2/2/00121 


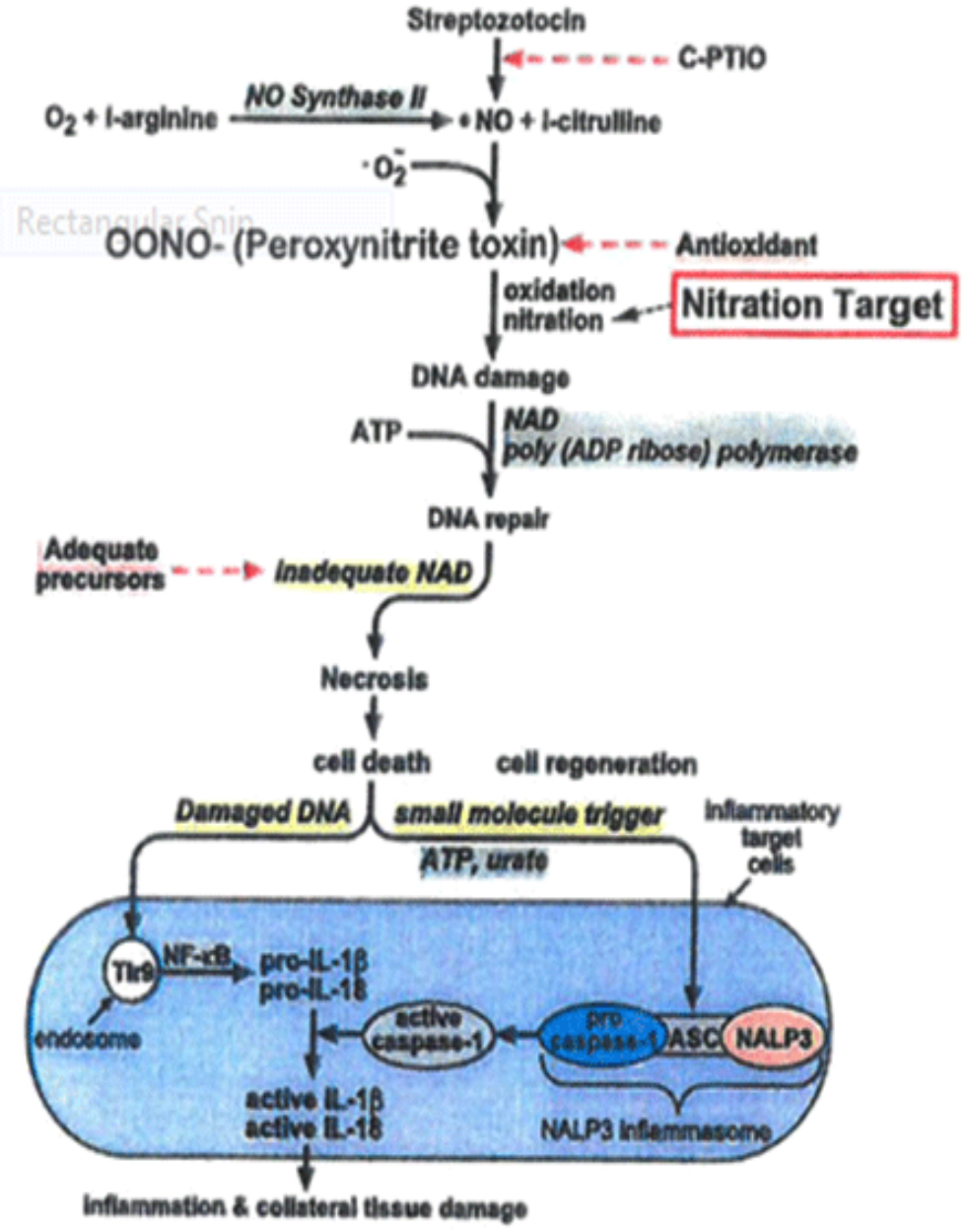

Figure 4: Roposed pathways generating excessive peroxynitrite and chronic inflammatory processes.

\section{Mechanisms and Biomarkers of Chronic Disease States and Biomarkers Produced From Oxidative/Nitrosation Damage Nitrosylation Nitration}

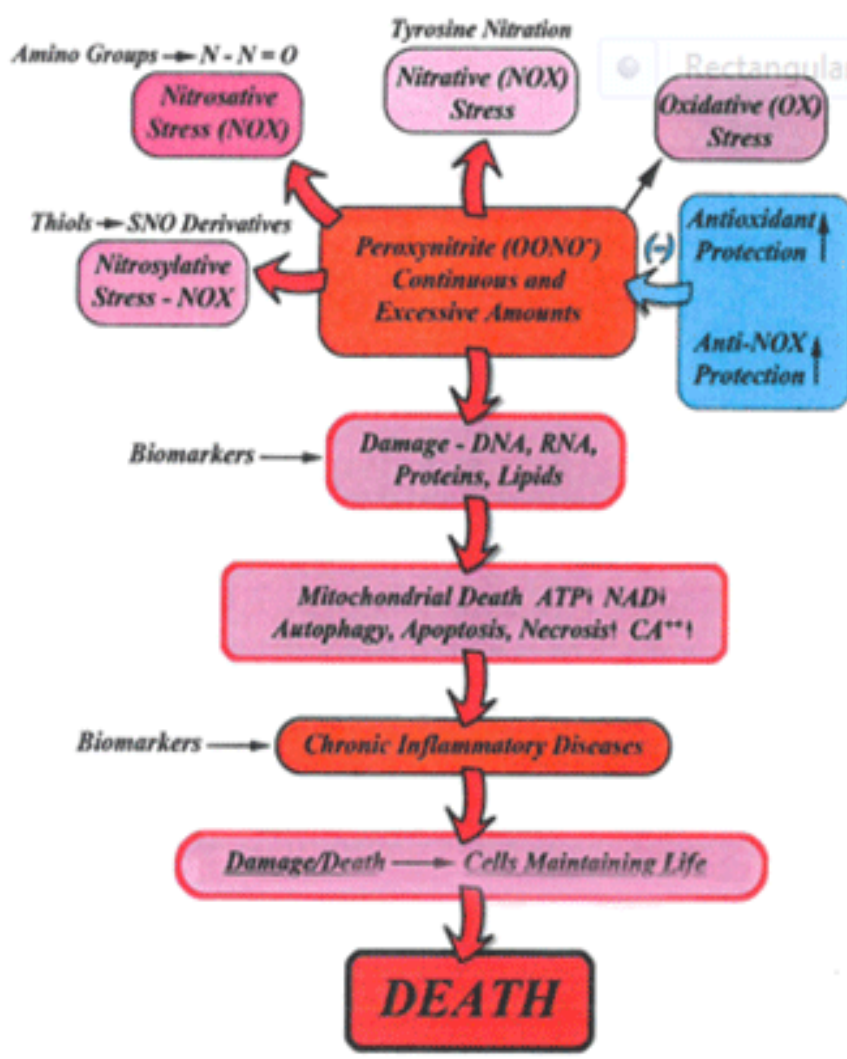

Figure 5: Mechanism and Biomarker's of chronic diseases which includes diabetes and Alzheimer's disease.

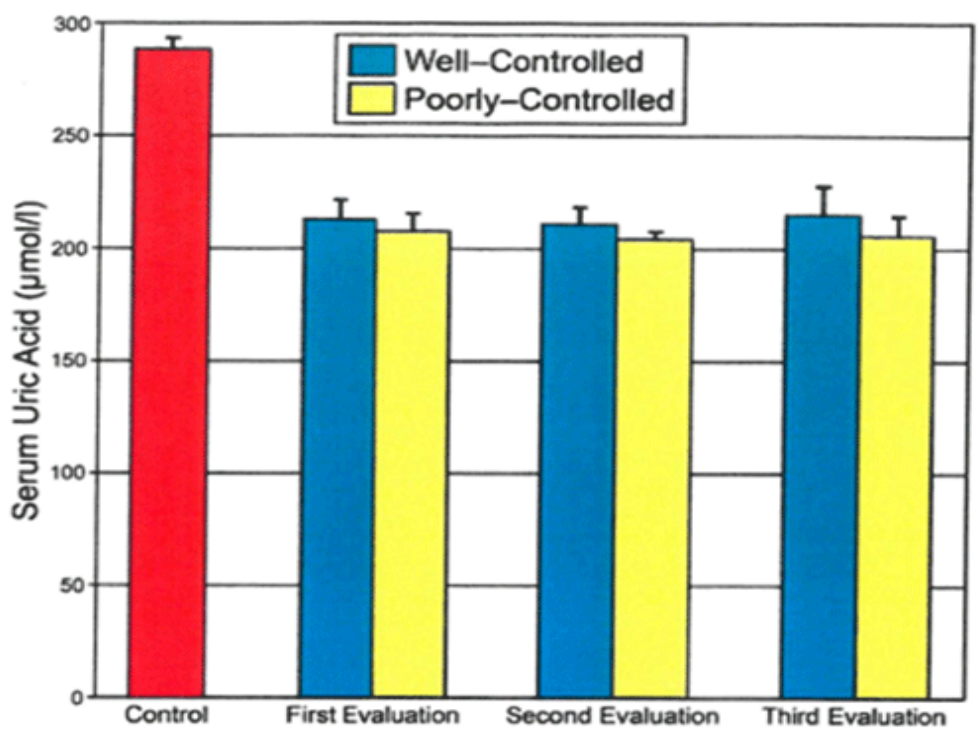

Figure 6: Graph depicting urate and glucose in the serum of 35 children with early type 1 diabetes and non-diabetic controls measured over a three year period. Both tightly controlled glucose and poorly controlled glucose children displayed a $25 \%$ decrease inn urate values compared to controls each of the 3 years measured from the earliest detection of diabetes1. It is well known that urate can destroy peroxynitrite and this depletes urate values. Whether the blood glucose is well controlled or not urate values in diabetic children with diabetes are constantly depleted. This indicates that the insulin treatment given to these children has little effect on basal levels of peroxynitrite damage.

is fairly specific, in that it reacts only with strong oxidizer/nitrators like peroxynitrite or hydrogen peroxide in the presence of horseradish peroxidase which is similar to peroxynitrite as a strong oxidizer. Substances which inhibit peroxynitrite are peroxynitrite decomposition catalysts (heme compounds) and various phenols and antioxidants like vitamin C and the different forms of vitamin $\mathrm{E}$ eg. tocopherols and tocotrienols and strong antioxidants eg: urate which can be nitrated and/or oxidized. In addition, substances that are activated to be readily nitrated or can destroy peroxynitrite such are substances which can be nitrated, nitrosylated or nitrosated are generally effective inhibitors of peroxynitrite based L-012 luminescence. See a series of experiments with peroxynitrite and L-012 and with STZ and L-012 and inhibitors for examples. The same inhibitors inhibit in both luminescent systems eg SIN-1 or STZ (Figures 7-10).

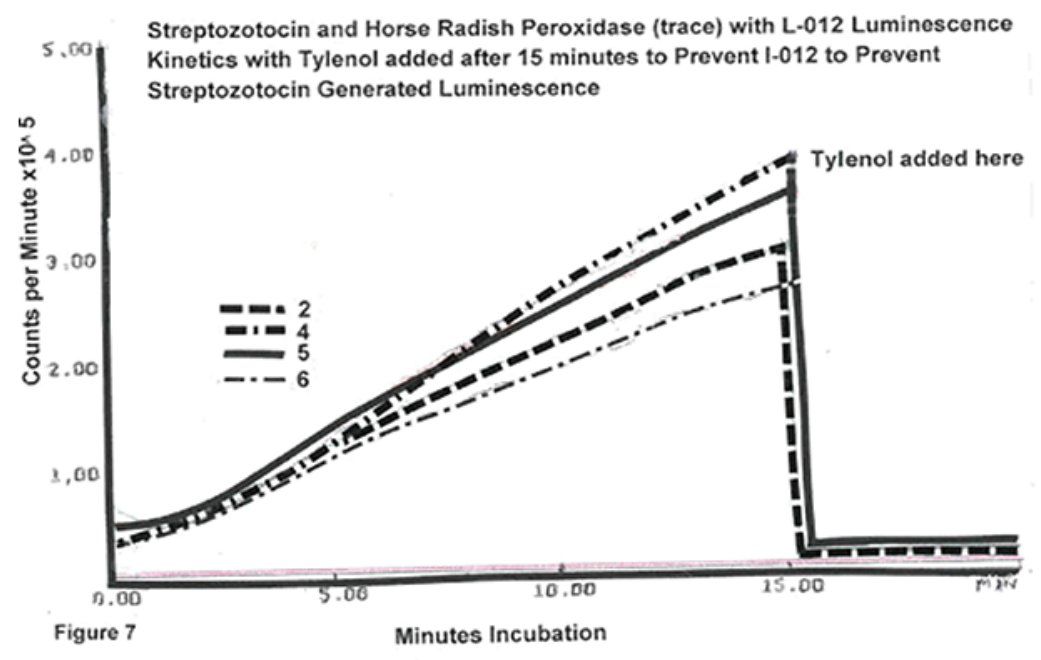

Figure 7: Streptozotocin generates peroxynitrite based- L-012 blue luminescence and if micromolar amounts of acetaminophen (Tylenol) are added once the reaction is started, the drug destroys the peroxynitrite shutting off the luminescence.

Citation: Dyke KV, Robart A, et al. (2017) Streptozotocin Generates Peroxynitrite Luminescence Which Reveals the Probable Cause of Diabetes 1,2 and 3 (Alzheimer's disease) Int J Open Access Ophthal 2(2):1-8. DOI: http://dx.doi.org/10.15226/2474-9249/2/2/00121 


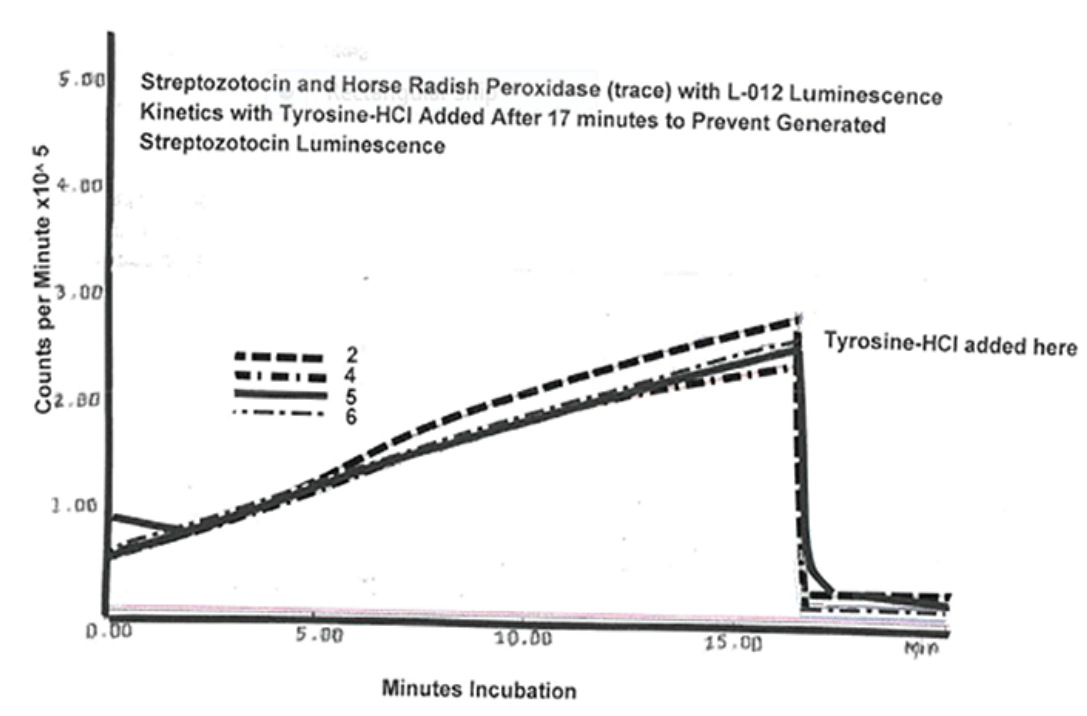

Figure 8: Streptozotocin generates L-012 based peroxynitrite based blue luminescence and if micromolar amounts of l-Tyrosine are added during the luminescent reaction, the luminescence is destroyed.

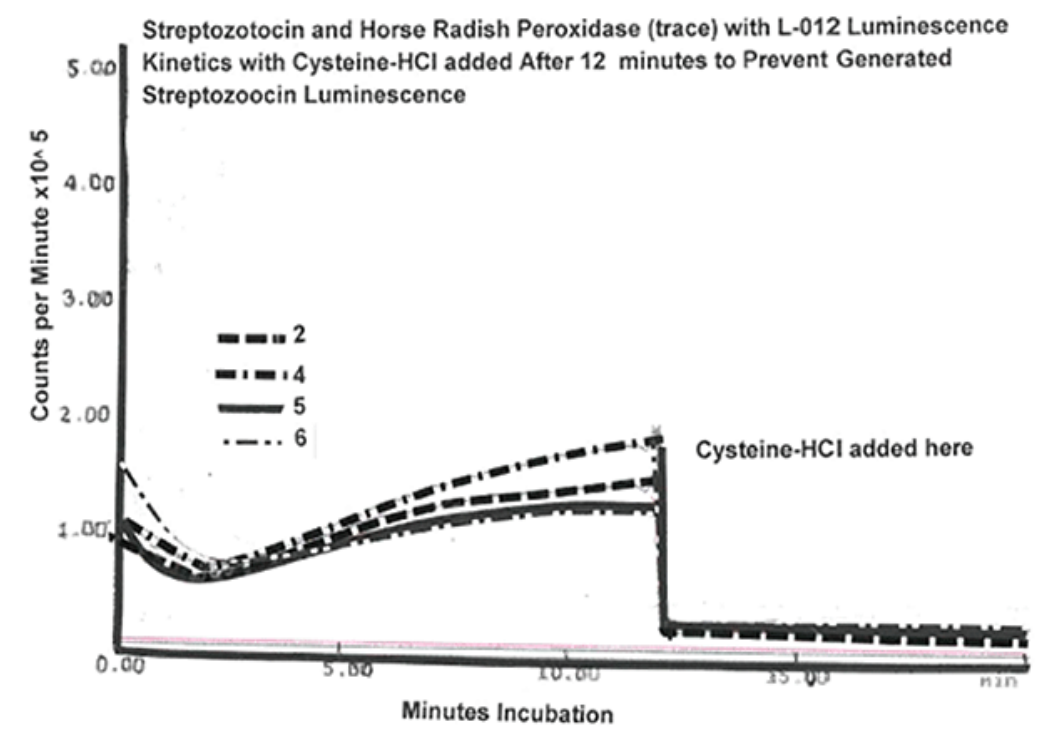

Figure 9: Streptozotocin generates peroxynitrite L-012 based luminescence and if micromolar amounts of L-cysteine hydrochloride are added during the luminescent reaction, the luminescence is destroyed.

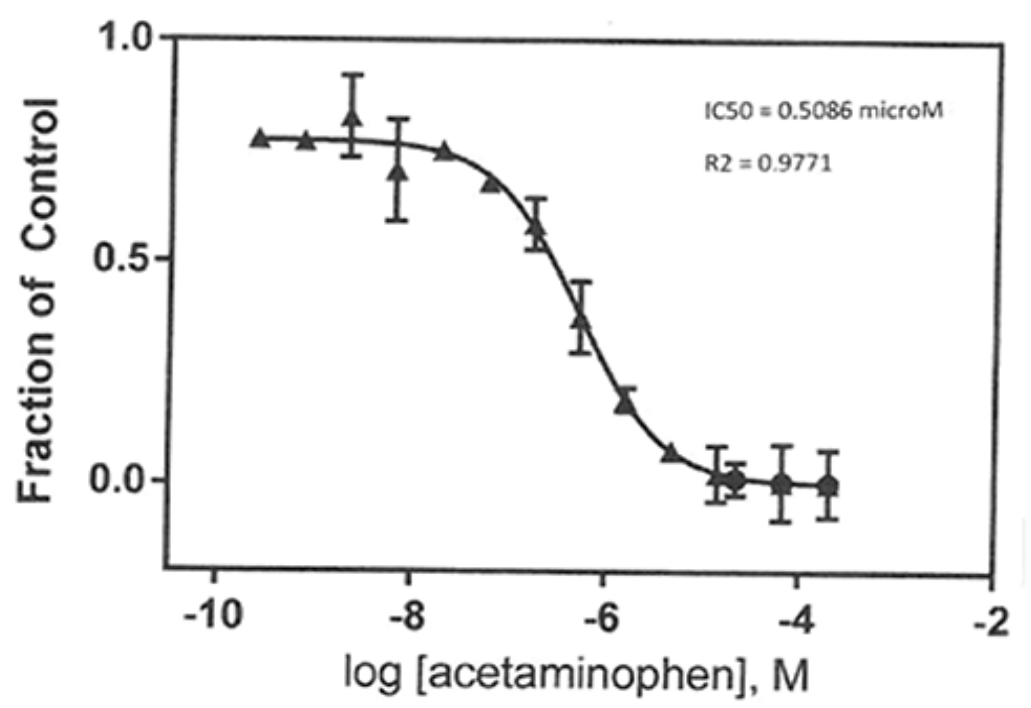

Figure 10: Dose-response of SIN-1 (peroxynitrite generator) and L-012 producing blue luminescence which is inhibited or destroyed a various micromolar doses of acetaminophen (Tylenol). The IC^50 is 0.51 micromolar.
Years ago, I wrote a very early article which suggested that peroxynitrite could cause Alzheimer's disease (19). I reasoned that plaques and tangles were unlikely to cause neuronal death, so to me it was much more likely that Alzheimer's disease was caused by an oxidizing acid. Macrophages in the brain (microglia) were a likely culprit and since I knew macrophages generated peroxynitrite anion of (peroxynitrous acid) which can oxidize luminol and cause blue luminescence. I reasoned that microglia (brain macrophages) could become activated generating excessive peroxynitrite which could damage neurons and cause the death of neurons as well creating the plaques and tangles in the hippocampus and elsewhere. After publication in 1997 it was shown that massive nitration of tyrosine proteins occurred in the areas of the brain in which Alzheimer's disease [20]. The only major substance known to exist in the body which could cause massive nitration of tyrosines of brain proteins is peroxynitrite produced mainly by macrophage's inducible NO synthase 2 and the macrophage membrane NADPH oxidase which produces the superoxide free radical -both reacting at diffusional speed to produce peroxynitrite anion (OON=O-). It can react with carbon dioxide further to produce peroxynitrite carbonate a excellent nitrator of phenols like tyrosine. Further, the anti-inflammatory reaction used by glucocorticoid steroids to inhibit acute inflammation namely histone deacetylase 2 (HDAC-2) (which normally cleaves the acetate groups from acetylated histones) is destroyed via excessive nitration of active site tyrosines emanating from excessive peroxynitrite in the active sites of HDAC-2 [21]. This allows inflammation to continue in a chronic state. Therefore, to control the chronic neuro-inflammatory state of Alzheimer's disease, excessive and continuous peroxynitrite needs to be controlled. Further, it needs to be controlled early in disease states - well before the final state of chronic inflammatory diseases which is death can occur.

Multiple scientists have used STZ via intra-articular injections into brains of normal mice to create an excellent mimic of Alzheimer's disease. In a few days post injection, the glucose mechanisms become depressed and insulin resistance in the brain occurs-just like PET scans of human brains (Figure 11). After a few months these mice develop plaques and tangles along with attention and memory deficits like Alzheimer's disease in humans. The neuroscientists could not understand why STZ could cause such a perfect Alzheimer's scenario. But, since we have clearly shown that STZ generates peroxynitrite and because the deoxyglucose portion of STZ brings the molecule to areas of high Glut 2 transporters -once inside, the key neuronal cells the methylated nitrosourea causes methylation and oxidation and nicking of DNA which activates the DNA repair system PARP (PolyADPribose polymerase) causing depletion of NADH and ATP from the mitochondria and causing necrosis of either beta cells or neurons. Surely this is why many neurological scientists call Alzheimer's disease diabetes 3 (see De La Monte and Wands 2008).

In Figure 12 we can observe the effects of streptozotocin upon double stranded DNA of a plasmid, but not on the DNA ladder. The plasmid DNA is a highly super coiled circle and the nicks of this DNA from streptozotocin cause the DNA to relax and so the broken DNA drags more slowly through the agarose. The ladder DNA does not appear to be damaged but the bases are methylated and oxidized by streptozotocin as shown previously by multiple investigators. These changes cannot be seen using our $2 \%$ 
agarose electrophoresis system.

\section{Diabetic Pathophysiology}

\section{Diabetic Cataracts and Retinopathy}

2. Diabetic Nephropathy-( Major Cause of Kidney Disease)

\section{Peripheral Artery Disease -Atherosclerosis; Hypertension; Endothelial/}

\section{Epithelial Cell Damage}

4. Heart disease-Mitochondrial Damage and Clots

\section{Erectile Dysfunction-Decreased Nitric Oxide Production Causing Guanyl-}

Cyclase Complex Degradation and Activation of Phosphodiesterase 5 with

Loss of Vasodilation

\section{Diabetic Neuropathy- Damage via NMDA Receptor Stimulation and Narcotic}

\section{Tolerance}

Figure 11: Pathology associated with diabetes. There are 6 major pathologies driven by peroxynitrite. They are: 1) diabetic retinopathy /cataracts 2) Diabetic nephropathy, 3) Peripheral artery disease, 4) heart disease and stroke, 5) Erectile dysfunction and 6) Diabetic neurpathy.

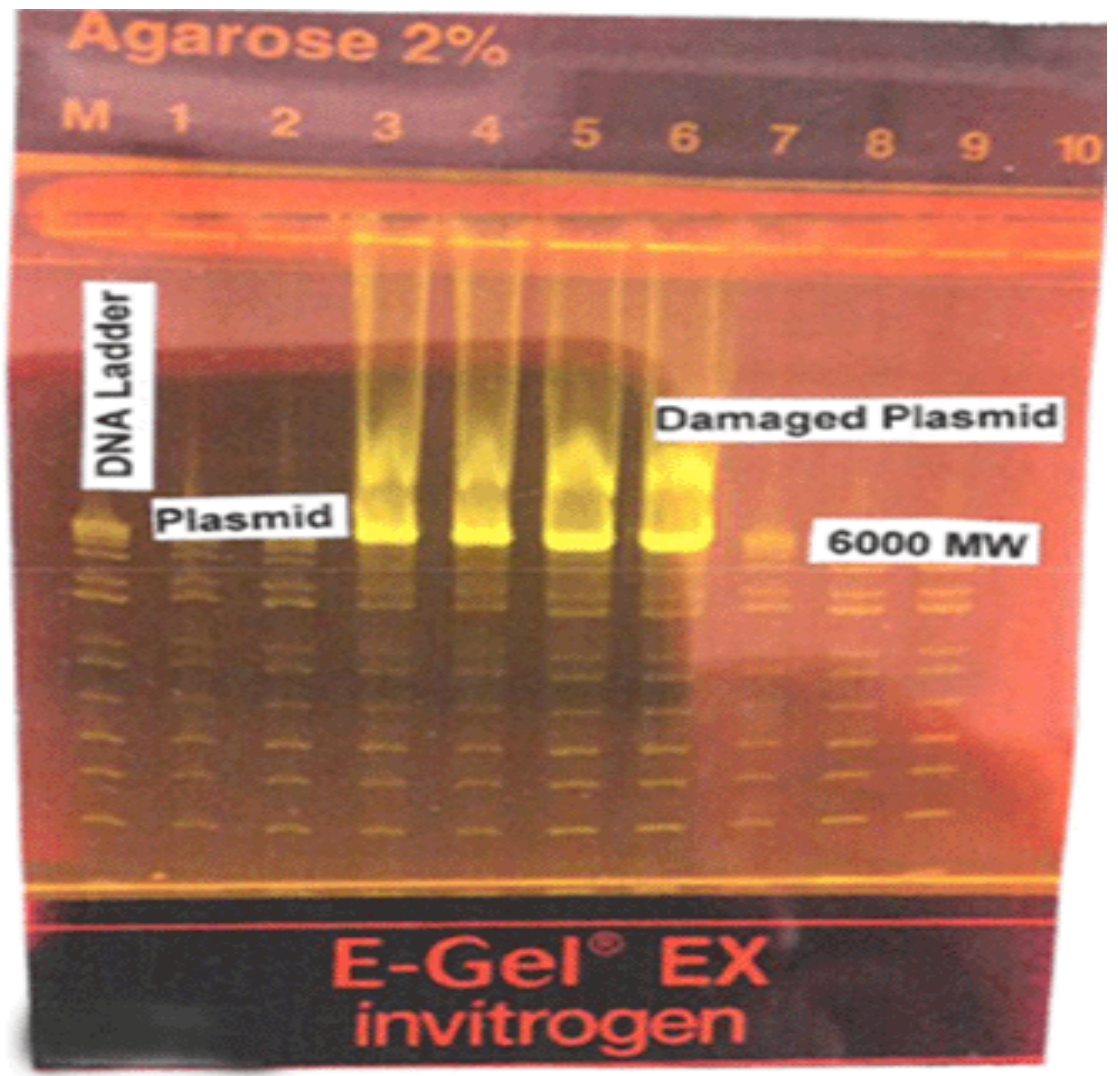

Figure 12: Electrophoresis of ladder DNA (Invitrogen) with top band at molecular weight $=6000$. and LUC plasmid DNA molecular weight $=6372 \mathrm{bps}$ both were treated with streptozotocin ((peroxynitrite source) at 37C for 30 minutes. Apparent damage appeared only to plasmid which produced two bands-one damaged which drags in the gel and the plasmid itself (lower area about 6000 molecular weight. This type of observation has made with peroxynitrite itself. The ladder DNA is actually oxidized and or methylated but this damage cannot be observed using fluorescent DNA assay.

Luminescence has been useful in the original discovery of nitric oxide and in its role in many diseases. It is now useful again in exposing the likely basis of both diabetes and likely Alzheimer's disease. We predict that it will be useful in the discovery of a practical solution in the prevention and a more complete treatment of these diseases and likely a cure for many of them- which we are presently studying. The final argument connecting diabetes and Alzheimer's disease was found in a comment found on the internet which is that: $80 \%$ of the people with Alzheimer's disease have insulin resistance or type 2 diabetes. The arguments drawn in this manuscript certainly indicate there is a clear scientific basis for this observation and there is a large amount of direct visual evidence using PET scans with18Fluorodeoxyglucose in early dementia that diabetes is the actual beginning of Alzheimer's disease and likely other dementias as well.

Summary: We and others demonstrate that streptozotocin (STZ) causes diabetes because the sugar portion of the molecule directs it to the GLUT2 transporters which concentrate in the beta cells of the pancreas which generates the oxidizing and nitrating molecule peroxynitrite (OONO-). This chemical damage kills the beta cell since OONO or its carbon dioxide derivative damages DNA, RNA, protein and lipids in the beta cells causing death of mitochondria and hence cell death. Interference with the production or the reaction of peroxynitrite can prevent diabetes. STZ when injected intra-articularly in the brain of a normal mouse creates the same scenario as early diabetes in the brain - namely decreased utilization of glucose, insulin resistance, but eventually after months post STZ injections- plaques and tangles with dementia occur. Likely, this is the cause of a neuro-inflammatory disease whose damage to the brain has many similarities to pathology of diabetes 1 and 2 . No wonder many physicians and scientists are calling

Alzheimer's disease diabetes 3. Further it is thought that antidiabetics could be useful in Alzheimer's disease but it will likely be found that both antidiabetic drugs and peroxynitrite targets will be needed early and continuously to prevent and control both major diseases .

\section{Acknowledgement}

Drs. Werner Geldenhuys and Aaron Robart of West Virginia University Medical School who were most helpful in this research.

\section{References}

1. Rakieten N, Rakieten M, Nadkarni Mv. Studies on the Diabetic Action of Streptozotocin (NSC 37917). Cancer Chemother Rep. 1963;29:91-98

2. Wu J, Yan L-J. Streptozotocin-induced type 1 diabetes in rodents as a model for studying mitochondrial mechanisms of diabetic $\beta$ cell glucotoxicity. Diabetes Metab Syndr Obes. 2015;8:181-188. doi: 10.2147/DMSO.S82272

3. Knox Van Dyke, Erica Ghareeb, Mark Van Dyke, Chris Van Dyke, Michael Gunther, David H Van Thiel. Does Nitric Oxide (NO) and Superoxide (.02-) cause Type 2 Diabetes and can it be Prevented? Biology and Medicine. 2014;6(1). doi: 10.4172/0974-8369.1000195

4. Van Dyke K, Ghareeb E, Van Dyke M, Sosa A, Hoeldtke RD, Van Thiel DH. Luminescence experiments involved in the mechanism of streptozotocin diabetes and cataract formation. Luminescence. 2008;23(6):386-391. doi: 10.1002/bio.1050

5. Van Dyke K, Jabbour N, Hoeldtke R, Van Dyke C, Van Dyke M. Oxidative/nitrosative stresses trigger type 1 diabetes: preventable in streptozotocin rats and detectable in human disease. Ann N.Y. Acad. Sci. 2010;1203:138-145. doi: 10.1111/j.17496632.2010.05563.x

6. Julius U, Drel VR, Grässler J, Obrosova IG. Nitrosylated Proteins in Monocytes as a Marker 
of Oxidative Stress in Diabetic Subjects with Microangiopathy. Exp. Clin. Endocrinol. Diabetes. 2009;117(2):72-77. doi: 10.1055/s-2008-1078710

7. Andersson DA, Filipović MR, Gentry C, Eberhardt M, Vastani N, Leffler A, et al. Streptozotocin stimulates the Ion Channel TRPA1 Directly: involvement of peroxynitrite. J Biol. Chem. 2015;290(24);15185-15196. doi: 10.1074/jbc.M115.644476

8. Tsuji A, Sakuri H. Generation of Nitric oxide from streptozotocin (STZ) in the presenve of Copper 2 and ascorbate: implications for the development of Streptozotocininduced diabetes. Biochem Biophys Res Commun. 1998;245(1):11-16. doi:10.1006/ bbrc.1998.8368

9. Chen Z, ZhongC. Decoding Alzheimer's Disease from perturbed cerebral glucose metabolism: Implications for diagnostic and therapeutic strategies. Prog Neurobiol. 2013 ;108:21-43. doi: 10.1016/j.pneurobio

10.Brown RK, Bohnen NI, Wong KK, Minoshima S, Frey KA. Brain PET in Suspected Dementia: Patterns of Altered FDG Metabolism. Radiographics. 2014;34(3):684-701. doi: $10.1148 / \mathrm{rg} .343135065$

11.Kamat PK. Streptozotocin induced Alzheimer's like changes and the underlying neural degeneration and regeneration mechanisms. Neural Regen. Res. 2015;10(7):1050 1052. doi: 10.4103/1673-5374.160076

12.Mehla J, Pahuja M, Gupta YK. Streptozotocin -induced sporadic Alzheimer's disease: selection of appropriate dose. 2013,33(1);17-21. doi: 10.3233/JAD-2012-120958

13.Kimura N. Diabetes Mellitus Induces Alzheimer's disease Pathology: Histopathological Eviddence from Animal Models. Int J Mol Sci. 2016;17(4):503. doi: 10.3390/ ijms17040503
14.Lecanu L, Papadopoulous V. Modeling Alzheimer's Disease with non-transgenic rat models. Alzheimers Res Ther. 2013;5(3):17. doi: 10.1186/alzrt171

15.Van Dyke K, Ghareeb E, Van Dyke M, Van Thiel DH. Ultrasensitive peroxynitrite based luminescence with L-012 as a screening system for anntioxidative/nitrosative substances eg Tylenol, 4 hydroxy Tenmpol , quercetin and carboxy-PTIO, Luminesence. 2007;22(4):267-274.

16. Van Dyke K, McConnell P, Marquardt L. Green tea polyphenols markedly inhibit luminol dependent chemiluminescence activated by peroxynitrite or SIN-1. Luminescence. 2000;15(1):37-43.

17.Statler K. Peroxynitrite-Driven mechanisms in Diabetes and Insulin Resistance-the Latest Advances, Curr. Med. Chem. 2011;18(2):280-290.

18.Dyke KV. New Concepts in Prevention and Treatment of Diabetes 1 and 2. Translational Medicine (Sunnyvale) 2016;6(3):179. doi: 10.4172/2161-1025.1000179

19.Van Dyke K. The possible role of peroxynitrite in Alzheimer's disease: A simple hypothesis that could be tested more thoroughly. Med Hypotheses. 1997;48(5);375380.

20.Mark A.S, Peggy LRH, Lawrence MS, Joseph SB, George P. Widespread peroxynitrite mediated damage in Alzheimer's disease. J. of Neuroscience. 1997;17(8):2653-2657.

21.Barnes PJ. How corticosteroids control inflammation: Quintiles Prize lecture 2005. British J. pharmacol. 2006;148(3):245-254. 